\title{
First report of a fatal autochthonous canine Angiostrongylus vasorum infection in Belgium
}

\author{
Sandra Jolly ${ }^{\mathrm{b}}$, Luc Poncelet ${ }^{\mathrm{c}}$, Laetitia Lempereur ${ }^{\mathrm{a}}$, Yannick Caron ${ }^{\mathrm{a}}$, Calixte Bayrou ${ }^{\mathrm{b}}$, Dominique Cassart ${ }^{\mathrm{b}}$, \\ Felix Grimm ${ }^{\mathrm{d}}$, Bertrand Losson ${ }^{\mathrm{a}, *}$

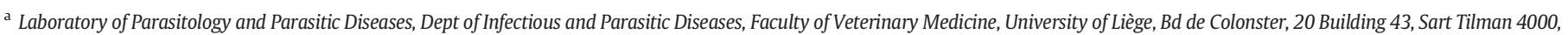 \\ Liège, Belgium \\ b Laboratory of Pathology, Dept of Morphology and Pathology, Faculty of Veterinary Medicine, University of Liège, Bd de Colonster, 20 Building 43, Sart Tilman 4000, Liège, Belgium \\ c Laboratory of Anatomy, Biomechanics and Organogenesis, Faculty of Medicine, Free University of Brussels, Route de Lennik 808,1070 Brussels, Belgium \\ d Institute of Parasitology, Vetsuisse Faculty, University of Zürich, Winterthurstrasse 266a, Zürich, Switzerland
}

\section{A R T I C L E I N F O}

Article history:

Received 19 May 2014

Received in revised form 16 October 2014

Accepted 21 October 2014

Available online 29 October 2014

\section{Keywords:}

Angiostrongylosis

Angiostrongylus vasorum

Dog

Belgium

\begin{abstract}
A B S T R A C T
Canine angiostrongylosis is considered as an emergent disease in Europe and Canada. A fatal case of Angiostrongylus vasorum infection is described in a four and a half month old puppy born in Belgium. The dog was presented with marked neurological disorders, body weight loss, a profound weakness and mild respiratory signs. The dog was given antibiotics and mucolytic compounds with very little improvement and consequently was referred to a specialist for additional examinations. As the general condition of the dog was rapidly declining, the animal was euthanized shortly after on its owners' request and a necropsy was carried out. Extensive gross pulmonary lesions were observed and histopathological examination revealed the presence of numerous larvae with morphology compatible with A. vasorum. Larvae were also found in the product of a bronchoalveolar lavage but fecal material was not examined. The presence of $A$. vasorum circulating serum antigen was demonstrated through ELISA; additionally an A. vasorum specific PCR was performed on brain material and yielded a positive result. This case confirms that the clinical diagnosis of canine angiostrongylosis can be very challenging especially when respiratory signs are absent or very mild such in the present case. This is the first reported case of canine angiostrongylosis in Belgium.
\end{abstract}

(c) 2014 Elsevier Ireland Ltd. All rights reserved.

\section{Introduction}

Canine angiostrongylosis is a gastropod borne helminthic infection which was first reported in France in the 1800's. Patent infections were also observed in red fox, wolf and European badger [1-4]. In Europe, the red fox is considered as the main reservoir host [5]. In dog, distinct isolated endemic foci were reported during the last century in south-western France, Ireland, Switzerland, Southeast England and Wales, and Denmark. Canine angiostrongylosis is clearly emerging both in North America [6] and Europe [7] where it was recently further recorded in Greece [8], The Netherlands [9], different parts of Germany [10], Italy [11], Slovakia [12] Poland [13] and Hungary [14]. In temperate Western Europe climatic conditions are highly suitable to the completion of Angiostrongylus vasorum life cycle and Belgium is part of a vast area characterized by its high predicted suitability for parasite transmission [15]. However, canine angiostrongylosis has not been recorded so far in this country. The present case report describes a fatal case of

\footnotetext{
* Corresponding author. Tel.: + 32436640 90; fax: + 3243664097

E-mail address: blosson@ulg.ac.be (B. Losson).
}

A. vasorum infection in a puppy born in Belgium and without travel history.

\section{Clinical presentation and post mortem findings}

A four and a half month old male Border Collie born in Belgium near the city of Liège was presented in November 2013 to a local veterinarian for apathy and poor growth ( $14 \mathrm{~kg}$; body score $2 / 5$ ). A clinical examination revealed a very moderate cough and respiratory noises suggestive of bronchitis. Body temperature was within normal range. The dog was given antibiotics (amoxicilline + clavulanic acid) and a mucolytic compound (carbocysteine). After a slight short-lived improvement the dog was re-examined for a severe loss of appetite and weakness. There were no other digestive signs but the dog presented obvious neurological signs and was referred as an emergency to a specialist. The general examination was judged within normal limits. It was slightly dull but responsive. Cranial nerve examination was normal. The dog displayed a severe ataxia in the hind limbs with occasional hypermetria. However, neither wide-based posture nor intention tremor was observed. A much more subtle ataxia of the thoracic limb, trunk and head movements was noted. In the hind limbs, placement reflexes 
were delayed, muscle tone was increased and stretch reflexes were exaggerated. Putative major lesion localization was spinal cord T2-L3 segments though multifocal or diffuse involvements were still considered; infectious/inflammatory causes were viewed as most likely.

A blood sample was collected for Toxoplasma gondii and Neospora caninum antibody search; muscle creatine kinase was also determined since concurrent muscle and nervous tissue involvement may occur with protozoan infections in this age class. A sample of cerebrospinal fluid was collected from the lumbar cistern under medethomidine/ thiopenthal anesthesia.

$T$. gondii and $N$. caninum serology yielded negative results whereas creatine kinase was within physiological range. Cerebrospinal fluid, red and white cell counts and total protein level were all within physiological range. A PCR for distemper virus was carried out on the cerebrospinal fluid sample and gave a negative result.

While waiting for the analysis results, a treatment was attempted immediately with clindamycin (Antirobe ${ }^{\mathrm{TM}}$ ), $300 \mathrm{mg} / \mathrm{d}$ orally and methylprednisolone (Moderin ${ }^{\mathrm{TM}}$ ) $12 \mathrm{mg} / \mathrm{d}$ orally.

Three days later the clinical condition of the dog had worsened (ataxia of the four limbs but more severe in the hind limbs, hypertonicity, hyperreflexivity, pain at manual mobilization) and the dog was humanely euthanized at its owner's request. Necropsy was performed at the Faculty of Veterinary Medicine, University of Liège.

Post mortem examination revealed multifocal interstitial pneumonia with consolidated areas and congestion. Right ventricular eccentric hypertrophy with atrial dilatation was found. These observations were associated with hepatic passive congestion, suggesting a congestive right heart failure. There were no gross lesions after opening the skull and cervical spinal canal.

Microscopic examination showed an extensive chronic interstitial verminous pneumonia in which fibroplasia predominated. Numerous larvae and eggs were embedded in fibrous inflammatory tissue with mononuclear cells, mostly macrophages. Vascular sclerosis was observed. First-stage larvae (L1), with a size and morphology compatible with $A$. vasorum, were observed in the larger airways (Figs. 1 and 2). However, Crenosoma vulpis was considered in the differential diagnosis.

The brain was cut into 5 sections. Only a small and multifocal perivascular infiltrate of lymphocytes was observed in the leptomeninges. The cervical spinal cord was not microscopically investigated. A piece of unfixed brain material was frozen for PCR examination.

A serum sample was sent to the Institute of Parasitology, University of Zurich and checked for the presence of A. vasorum specific circulating antigen; the sample gave a strong positive reaction (OD/cut off ratio: 5.6).

DNA was isolated from $25 \mathrm{mg}$ ethanol fixed brain material (QIAamp DNA Mini Kit, QIAGEN GmbH, Hilden, Germany) and a highly specific

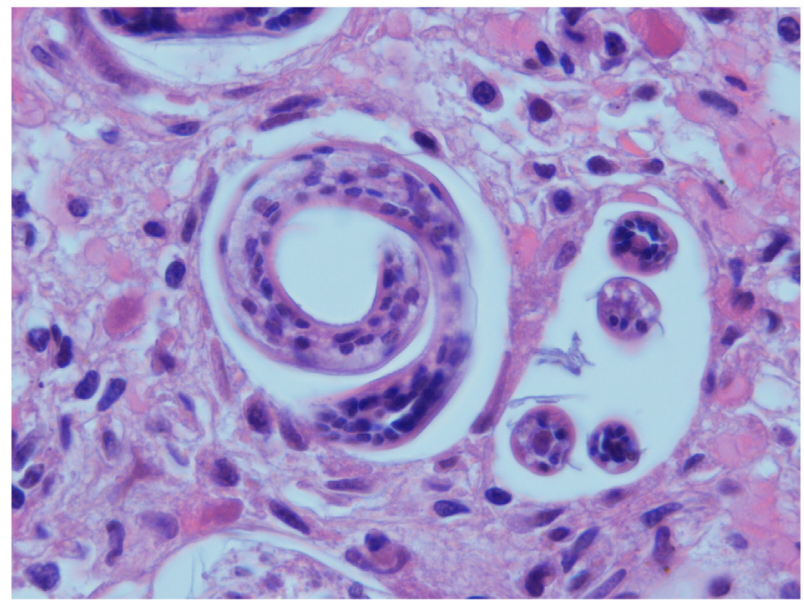

Fig. 1. First-stage larvae (L1), with a size and morphology compatible with Angiostrongylus vasorum, were observed in the larger airways.

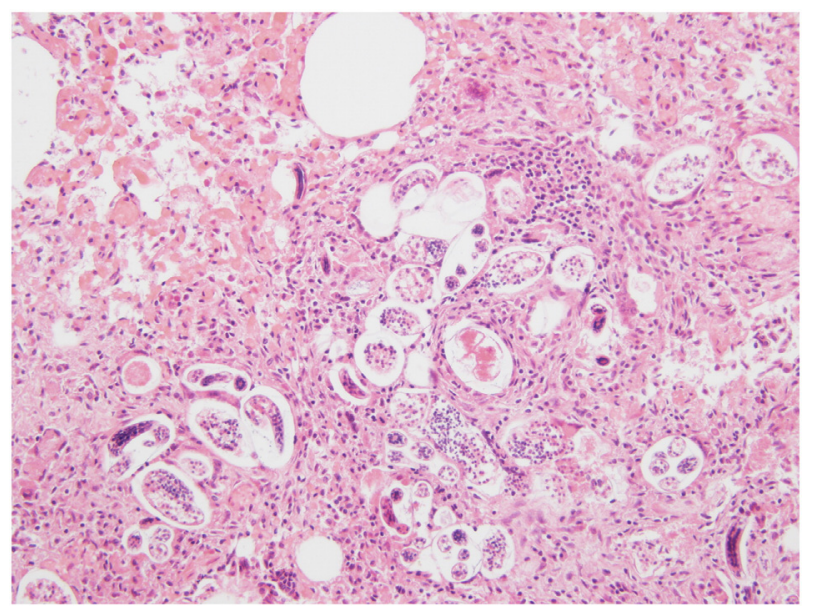

Fig. 2. Chronic interstitial pneumonia with prominent fibrosis. A granuloma around eggs and larvae of Angiostrongylus vasorum is associated with pneumocyte proliferation and lymphocyte/macrophage infiltration.

fluorescent probe-based, real time PCR assay targeting a 121 bp long fragment of the ITS2 region [16] of A. vasorum was positive (Ct 29.4).

\section{Discussion}

This is the first report of canine angiostrongylosis in Belgium. The young age of the dog and the fact that it was born in Belgium indicate that the infection was acquired locally through the ingestion of infected slugs or their secretions. The ingestion of paratenic hosts such as frogs can also play a role but $A$. vasorum prepatent period (47-49 days in experimentally infected dogs [17]) and the age of the puppy when first examined ( 4 months and a half) make this mode of infection very unlikely in the present case. A definitive diagnosis of angiostrongylosis was established post mortem through histopathology and the observation of larvae in the lung parenchyma. This was further confirmed via the detection of specific circulating antigens using a sandwich ELISA and PCR on brain material. The ELISA test is based on a monoclonal antibody ( $m A b$ Av 56/1/2) and on polyclonal rabbit antibodies directed against $A$. vasorum adult excretory/secretory antigens. The sensitivity and specificity of this test reached $95.7 \%$ and $94.0 \%$ respectively [18].

Often A. vasorum is responsible for subclinical infections or mild to moderate respiratory signs [19]. However, severe signs are increasingly reported such as exercise intolerance, bleeding diatheses, heart failure, neurological disorders and even sudden death [20]. The present case was characterized by major neurological signs associated with very mild respiratory signs. A minor proportion (approximately 4\%) of A. vasorum infected dogs presents neurological disorders [20-22]. This central nervous system implication would result primarily from hemorrhages in the CNS induced by coagulation disturbances or alternatively by migrating larvae $[22,23]$. However, larvae induced lesions and clinical bleeding disorders were not observed. In the present case a timely diagnosis based on a recently developed serological in-clinic diagnostic tool [24] and treatment with appropriate drugs were confounded by the age of the dog, the clinical picture and the absence of records for Belgium. It is interesting to point out that very mild respiratory signs were observed in this patient; however at post mortem severe gross lesions of pneumonia were observed. The small adult worms probably were missed during necropsy. In a recent large sero-epidemiological survey conducted in Western Germany combining the detection of A. vasorum antigen and specific antibodies [25], 0.3\%, 0.5\% and 2.25\% of the samples were found positive using both ELISAs, antigen-ELISA and antibody-ELISA respectively. Some of the western federal states participating to this survey have a border with Belgium and the city of Liège is only approximately $35 \mathrm{~km}$ far from the German border. Thus, the presence of A. vasorum in this area was to be expected. Besides, 
recent unpublished observations by local vets reporting chronic respiratory signs associated with a lung pattern compatible with angiostrongylosis suggest that the area is endemic for A. vasorum. The red fox (Vulpes vulpes) could be responsible for the introduction and spreading of the parasite as recently described in The Netherlands [26]. Clearly, additional studies in both final and intermediate hosts as well as proper information of practitioners regarding diagnosis and treatments are needed taking into account that highly effective drugs such as fenbendazole [27], moxidectin $[27,28]$ and milbemycin oxime $[29,30]$ are widely available.

\section{Acknowledgments}

We would like to thank Drs Boland and Carlier who provided useful clinical information on this case.

\section{References}

[1] Ferdushy T, Hasan MT. Angiostrongylus vasorum: the "French Heartworm". Parasitol Res 2010;107:765-71.

[2] Guilhon J. Recherchessur le cycle évolutif du Strongle des vaisseaux du chien. Bull Acad Vét 1963;36:431-42.

[3] Segovia JM, Torres J, Miquel J, Llaneza L, Feliu C. Helminths in the wolf Canis lupus from north-western Spain. J Helminthol 2001;75:183-92.

[4] Torres A, Pantchev N, Vrhovec MG, Bauer C, Hermosilla C. Lungworm infections (Angiostrongylus vasorum, Crenosoma vulpis, Aelurostrongylus abstrusus) in dogs and cats in Germany and Denmark in 2003-2007. Vet Parasitol 2008;159:175-80.

[5] Bolt G, Monrad J, Henriksen P, Dietz HH, Koch J, Bindseil E, et al. The fox (Vulpes vulpes) as a reservoir for canine Angiostrongylosis in Denmark. Field survey and experimental infections. Acta Vet Scand 1992;33:357-62.

[6] Conboy GA. Canine angiostrongylosis: the French heartworm: an emerging threat in North America. Vet Parasitol 2011:176:382-9.

[7] Helm JR, Morgan ER, Jackson MW, Wotton P, Bell R. Canine angiostrongylosis: an emerging disease in Europe. J Vet Emerg Crit Care (San Antonio) 2010;20:98-109.

[8] Papazahariadou M, Founta A, Papadopoulos E, Chliounakis S, Antoniadou-Sotiriadou K, Theodorides Y. Gastrointestinal parasites of shepherd and hunting dogs in the Serres Prefecture, Northern Greece. Vet Parasitol 2007;148:170-3.

[9] van Doorn DC, van de Sande AH, Nijsse ER, Eysker M, Ploeger HW. Autochthonous Angiostrongylus vasorum infection in dogs in The Netherlands. Vet Parasitol 2009; 162:163-6.

[10] Barutzki D, Schaper R. Natural infections of Angiostrongylus vasorum and Crenosoma vulpis in dogs in Germany (2007-2009). Parasitol Res 2009;105(Suppl. 1):S39-48.

[11] Guardone L, Schnyder M, Macchioni F, Deplazes P, Magi M. Serological detection of circulating Angiostrongylus vasorum antigen and specific antibodies in dogs from central and northern Italy. Vet Parasitol 2013;192:192-8.

[12] Hurnikova Z, Miterpakova M, Mandelik R. First autochthonous case of canine Angiostrongylus vasorum in Slovakia. Parasitol Res 2013;112:3505-8.
[13] Schnyder M, Schaper R, Pantchev N, Kowalska D, Szwedko A, Deplazes P. Serological detection of circulating Angiostrongylus vasorum antigen- and parasite-specific antibodies in dogs from Poland. Parasitol Res 2013;112(Suppl. 1):109-17.

[14] Majoros G, Fukar O, Farkas R. Autochthonous infection of dogs and slugs with Angiostrongylus vasorum in Hungary. Vet Parasitol 2010;174:351-4.

[15] Morgan ER, Jefferies R, Krajewski M, Ward P, Shaw SE. Canine pulmonary angiostrongylosis: the influence of climate on parasite distribution. Parasitol Int 2009; 58:406-10.

[16] Jefferies R, Morgan ER, Helm J, Robinson M, Shaw SE. Improved detection of canine Angiostrongylus vasorum infection using real-time PCR and indirect ELISA. Parasitol Res 2011;109:1577-83.

[17] Schnyder M, Fahrion A, Riond B, Ossent P, Webster P, Kranjc A, et al. Clinical, laboratory and pathological findings in dogs experimentally infected with Angiostrongylus vasorum. Parasitol Res 2010;107:1471-80.

[18] Schnyder M, Tanner I, Webster P, Barutzki D, Deplazes P. An ELISA for sensitive and specific detection of circulating antigen of Angiostrongylus vasorum in serum samples of naturally and experimentally infected dogs. Vet Parasitol 2011;179:152-8.

[19] Paradies P, Schnyder M, Capogna A, Lia RP, Sasanelli M. Canine angiostrongylosis in naturally infected dogs: clinical approach and monitoring of infection after treatment. Scientific World Journal 2013. http://dx.doi.org/10.1155/2013/702056 [Article ID 702056, 8 pages, 2013].

[20] Koch J, Willesen JL. Canine pulmonary angiostrongylosis: an update. Vet J 2009;179: 348-59.

[21] Chapman PS, Boag AK, Guitian J, Boswood A. Angiostrongylus vasorum infection in 23 dogs (1999-2002). J Small Anim Pract 2004;45:435-40.

[22] Gredal H, Willesen JL, Jensen HE, Nielsen OL, Kristensen AT, Koch J, et al. Acute neurological signs as the predominant clinical manifestation in four dogs with Angiostrongylus vasorum infections in Denmark. Acta Vet Scand 2011;53:43.

[23] Morgan ER, Shaw SE, Brennan SF, De Waal TD, Jones BR, Mulcahy G. Angiostrongylus vasorum: a real heartbreaker. Trends Parasitol 2005;21:49-51.

[24] Schnyder M, Stebler K, Naucke TJ, Lorentz S, Deplazes P. Evaluation of a rapid device for serological in-clinic diagnosis of canine angiostrongylosis. Parasit Vectors 2014; 7:72.

[25] Schnyder M, Schaper R, Bilbrough G, Morgan ER, Deplazes P. Seroepidemiological survey for canine angiostrongylosis in dogs from Germany and the UK using combined detection of Angiostrongylus vasorum antigen and specific antibodies. Parasitology 2013;140:1442-50.

[26] Franssen F, Nijss R, Mulder J, Cremers H, Dam C, Takumi K, et al. Increase in number of helminth species from Dutch red foxes over a 35-year period. Parasit Vectors 2014;7:166. http://dx.doi.org/10.1186/1756-3305-7-166 [2014 Apr 3].

[27] Willesen JL, Kristensen AT, Jensen AL, Heine J, Koch J. Efficacy and safety of imidacloprid/moxidectin spot-on solution and fenbendazole in the treatment of dogs naturally infected with Angisotrongylus vasorum (Baillet, 1866). Vet Parasitol 2007; 147:258-64.

[28] Schnyder M, Fahrion A, Ossent P, Kohler L, Webster P, Heine J, et al. Larvicidal effect of imidacloprid/moxidectin spot-on solution in dogs experimentally inoculated with Angiostrongylus vasorum. Vet Parasitol 2009;166:326-32.

[29] Bohm C, Schnyder M, Thamsborg SM, Thompson CM, Trout C, Wolken S, et al. Assessment of the combination of spinosad and milbemycin oxime in preventing the development of canine Angiostrongylus vasorum infections. Vet Parasitol 2014; 199:272-7.

[30] Conboy G. Natural infections of Crenosoma vulpis and Angiostrongylus vasorum in Atlantic Canada and their treatment with milbemycin oxime. Vet Rec 2004;155: $16-8$. 